\title{
Modulation of granulocyte responses in three- spined sticklebacks Gasterosteus aculeatus infected with the tapeworm Schistocephalus solidus
}

\author{
J. P. Scharsack ${ }^{1,2}$, M. Kalbe ${ }^{1, *}$, R. Derner $^{1}$, J. Kurtz ${ }^{1}$, M. Milinski ${ }^{1}$ \\ ${ }^{1}$ Max Planck Institute of Limnology, Department of Evolutionary Ecology, August-Thienemann-Strasse 2, \\ 24306 Plön, Germany \\ ${ }^{2}$ Present address: Biology and Immunology Group, Wageningen University, PO Box 338, 6700 AH Wageningen, \\ The Netherlands
}

\begin{abstract}
Leukocytes isolated from the head kidney and peripheral blood of 3-spined sticklebacks Gasterosteus aculeatus L. were analysed by means of flow cytometry during infection with the tapeworm Schistocephalus solidus (Müller, 1776). Although parasites increased their body weight continuously throughout the observation period (98 d), proportions of granulocytes increased in blood and head kidney only up to Day 63 post-infection (p.i.). Thereafter, declining proportions of granulocytes were observed in both organs. Thus the relative decrease in granulocyte number was not correlated to a decline in the parasitic load of the fish. To investigate a possible modulatory impact of $S$. solidus on granulocyte function, head kidney leukocytes were isolated at times before Day 63 p.i. and tested in vitro for their capacity to produce reactive oxygen species (ROS). Head kidney leukocytes from $S$. solidus-infected fish, analysed immediately after isolation (ex vivo, Day 40 p.i.), exhibited a higher ROS production when stimulated with phorbol myristate acetate (PMA), than leukocytes from naïve, sham-treated control fish and fish that had resisted or cleared the infection (exposed but not infected). The latter showed an increased spontaneous ROS production that was not correlated to the numbers of granulocytes present in the head kidney isolates. In infected sticklebacks, spontaneous and PMA-induced ROS production was significantly correlated with numbers of granulocytes present in the head kidney isolates, suggesting that elevated ROS production was due to higher numbers of responding cells rather than an increased capacity of single cells. In vitro, after cultivation for $4 \mathrm{~d}$ in the presence of pokeweed mitogen (PWM) or extracts from S. solidus, head kidney leukocytes from control fish showed an increased ROS production and phagocytic activity compared with nonstimulated control cultures. In contrast, head kidney leukocytes from infected fish isolated on Days 48 and 44 p.i., failed to respond to $S$. solidus antigens in vitro. During $S$. solidus infection, granulocyte mobilisation resulted in elevated numbers of these cells in head kidneys, but the lack of an in vitro response to $S$. solidus antigens indicates an in vivo priming of granulocytes by the parasite. These observations may reflect the ability of $S$. solidus to impair the host's immune response once the parasite is developing in the body cavity of $G$. aculeatus.
\end{abstract}

KEY WORDS: Gasterosteus aculeatus $\cdot$ Schistocephalus solidus $\cdot$ Granulocytes $\cdot$ Reactive oxygen species $\cdot$ Phagocytosis

Resale or republication not permitted without written consent of the publisher

\section{INTRODUCTION}

The plerocercoid larvae of the pseudophyllidean cestode Schistocephalus solidus are frequent parasites in the body cavity of the 3-spined stickleback throughout its geographical range (Arme \& Owen 1967). The infection is under investigation as a model of host- parasite interaction in studies on behaviour (Milinski 1984, 1985, 1990, Øverli et al. 2001), reproduction (Meakins 1974, McPhail \& Peacock 1983, Candolin \& Voigt 2001) and evolutionary ecology (Barber \& Ruxton 1998, Aeschlimann et al. 2000, Arnott et al. 2000, Binz et al. 2000, Reimchen \& Nosil 2001, Christen \& Milinski 2003, Kurtz et al. 2004). Yet little is known about 
immune mechanisms that may play a role in $S$. solidus infection of the stickleback.

Mobilisation and activation of granulocytes has been considered a significant part of the immune response of cyprinids (Hoole \& Arme 1983, Taylor \& Hoole 1993, 1995, Nie \& Hoole 2000) and rainbow trout (Sharp et al. 1992) to helminth parasites. Increased production of oxygen intermediates like nitric oxide (NO) and reactive oxygen species (ROS) occur (Whyte et al. 1990, Secombes \& Chappell 1996), indicating the functional importance of granulocyte and macrophage responses to helminths. Indeed, in vitro larvacidal activity of macrophages from rainbow trout to diplostomules of the eye fluke Diplostomum spathaceum has been demonstrated (Whyte et al. 1989). It is not yet clear whether large helminth parasites of fishes are killed by cellular responses in vivo (Secombes \& Chappell 1996). The fate of Schistocephalus solidus plerocercoids in fish species other than the natural host, the 3-spined stickleback Gasterosteus aculeatus, do however indicate that the plerocercoids can be harmed by fish immune defence. Growth of plerocercoids was much slower in 9-spined sticklebacks Pungitius pungitius, in which degenerative changes in the plerocercoids' tegument were detected (Orr et al. 1969). Heterotransplants of S. solidus from G. aculeatus to other species of fishes (Cottus gobio, Nemacheilus barbatula, Phoxinus phoxinus, Salmo trutta, Coregonus clupeoides, Perca fluviatilis, Rutilus rutilus, Esox lucius), including $P$. pungitius, died within 2 to $10 \mathrm{~d}$ of transfer, while homotransplants between G. aculeatus survived (Bråten 1966). Whether the parasites were actively destroyed by the immune system was not analysed. However these observations indicate that $S$. solidus plerocercoids may be able to suppress an effective immune response in G. aculeatus, but not in other fish species.

Information on the cellular immune response of stickleback against Schistocephalus solidus is still limited. Although encystment of $S$. solidus plerocercoids in the body cavity of infected sticklebacks was not observed (Arme \& Owen 1967), total white blood cell counts increased during $S$. solidus infection (Barber et al. 2001). Thus leukocytes are mobilised during $S$. solidus infection of stickleback, but the parasite may have evolved strategies of immuno-suppression or immuno-avoidance.

The present study investigated cellular responses of sticklebacks infected with Schistocephalus solidus. Leukocytes isolated from the peripheral blood and the head kidney of stickleback were analysed by means of flow cytometry for the frequency of leukocyte subsets and production of reactive oxygen species (ROS), and phagocytosis activity of head kidney leukocytes were analysed following in vitro stimulation with $S$. solidus extracts.

\section{MATERIALS AND METHODS}

\section{Propagation of Gasterosteus aculeatus and Schisto-} cephalus solidus. Both fish and tapeworms were laboratory-raised offspring of individuals originating from the Neustädter Binnenwasser (northern Germany), a brackish lagoon of the Baltic Sea. Sticklebacks were raised and kept in groups of 10 to 20 fish in 201 tanks with a continuous supply of aerated tap water at $18^{\circ} \mathrm{C}$ and a day:night rhythm of 16:8 h. During the first few weeks after hatching, fish were fed daily with Artemia sp. naupliae, and later 3 to 5 times per week with frozen mosquito larvae and occasionally frozen cladocerans ad libitum. Mature $S$. solidus plerocercoids were removed from the fish body-cavity aseptically and allowed to reproduce in an in vitro system modified from Smyth (1954), as described in Wedekind et al. (1998). Eggs released by the worms were washed and stored in tap water at $18^{\circ} \mathrm{C}$ in the dark. After $20 \mathrm{~d}$ incubation, hatching of coracidia was stimulated by light (Dubinina 1980).

Infection experiments. As first intermediate hosts, laboratory-bred copepods Macrocyclops albidus were each individually incubated with 6 Schistocephalus solidus coracidia, and screened for intensity of infection after 2 wk. Subsequently, individual sticklebacks were exposed to 3 copepods containing together a total of 4 procercoids in tanks with $500 \mathrm{ml}$ of water for $48 \mathrm{~h}$. Ingestion of the intermediate hosts was confirmed by subsequent filtration of the water and screening for remaining copepods. To follow up the development of $S$. solidus plerocercoids associated with expected changes in frequencies of leukocyte subpopulations, reflecting an immune response, 105 sticklebacks were exposed to infected copepods and kept in groups of 15 in separate tanks. All fish from individual tanks were sacrificed and sampled at set time points (see Fig. 2). Infection was monitored by measuring the fresh weight of $S$. solidus plerocercoids. Head kidney and peripheral blood leukocytes were isolated from infected fish. From the same stock, 20 fish that were not exposed to copepods were analysed as a Day 0 control. For in vitro analysis of leukocytes from $S$. solidus-infected sticklebacks, 36 sticklebacks were exposed to infected copepods and 18 fish were exposed to non-infected copepods (sham-exposed). Exposed fish were kept in groups of 6 in separate tanks until sampling. Head kidney leukocytes were collected at time points before the peak of granulocyte frequencies (see Fig. 2) from 12 fish exposed to infected copepods and 6 sham-exposed fish at 40,44 and $48 \mathrm{~d}$.

Preparation of Schistocephalus solidus extract. Plerocercoids of $S$. solidus, with an individual weight of 15 to $200 \mathrm{mg}$, were collected from laboratory-infected sticklebacks. Plerocercoids were washed with phos- 
phate-buffered saline (PBS) and frozen at $-20^{\circ} \mathrm{C}$ in PBS $\left(1 \mathrm{~g}\right.$ wet weight $\mathrm{ml}^{-1} \mathrm{PBS}, \mathrm{pH}$ 7.4). After thawing, the worms were chopped up with a scalpel and sonicated for $60 \mathrm{~s}$ on ice. The resulting suspension was centrifuged at $20800 \times g$ and $4^{\circ} \mathrm{C}$ for $15 \mathrm{~min}$. The supernatant was removed and $0.45 \mu \mathrm{m}$-filtered. Subsequently the protein content was determined colorimetrically using bovine serum albumin as a standard, and aliquots were stored at $-20^{\circ} \mathrm{C}$.

Culture media. Culture media for cell separation and cultivation and phosphate-buffered saline (PBS) were diluted with $10 \%(\mathrm{v} / \mathrm{v})$ distilled water to adjust their osmotic pressure according to stickleback serum osmolarity. For blood collection, diluted Leibovitz 15 (L-15) medium (Sigma Aldrich) with $50000 \mathrm{IU} \mathrm{l}^{-1}$ sodium heparin (Sigma-Aldrich) was used (heparinised medium). Washing procedures were conducted with diluted L-15 (wash medium). For cultivation experiments diluted L-15 was supplemented with $100000 \mathrm{IU} \mathrm{l}^{-1}$ penicillin, $100 \mathrm{mg} \mathrm{l}^{-1}$ streptomycin, $4 \mathrm{mmol} \mathrm{l}^{-1}$ L-glutamine, $7 \%(\mathrm{v} / \mathrm{v})$ foetal bovine serum (FBS; all chemicals: Sigma Aldrich) and 3\% (v/v) carp serum (culture medium). Carp serum was used as a source of piscine serum because of the limitations inherent in the preparation of sufficient amounts of stickleback serum. Carp serum was a pool of sera from 10 individual fish. It was heat-inactivated for $30 \mathrm{~min}$ at $56^{\circ} \mathrm{C}, 0.2 \mu \mathrm{m}$-filtered and stored at $-22^{\circ} \mathrm{C}$ until use.

Leukocyte isolation. Media and cells were kept on ice, and washing procedures were performed at $4^{\circ} \mathrm{C}$. Fish were anaesthetised by a blow on the head and killed by incision of the brain. Blood was collected into wells of 24-well plates prefilled with $0.5 \mathrm{ml}$ heparinised medium, after cutting off the tail of the fish. Peripheral blood leukocytes (PBL) were separated from erythrocytes by centrifugation $(30 \mathrm{~min}, 750 \times g)$ over Lymphoprep (1.077 $\mathrm{g} \mathrm{ml}^{-1}$, Nycomed) discontinuous density-gradient, as described by Miller \& McKinney (1994). Cell suspensions from head kidneys were prepared by forcing the tissues through a $40 \mu \mathrm{m}$ nylon screen (BD-Falcon, USA). Isolated PBL and head kidney leukocytes (HKL) were washed once with wash medium $(10 \mathrm{~min}, 550 \times \mathrm{g})$ and once with culture medium, and resuspended in cell-culture medium. Numbers of viable cells (exclusion of propidiumiodide-positive cells) were enumerated by means of flow cytometry.

Flow cytometry analysis. Suspensions of freshly isolated and of cultured PBL or HKL were analysed by flow cytometry (PAS III, Partec, Germany). All samples were supplemented with propidium iodide $\left(2 \mathrm{mg} \mathrm{l}^{-1}\right.$, Sigma Aldrich) to detect dead cells, and tubes were kept on ice until measurement. Forward- and sidescatter values (FSC/SSC characteristics) of at least 10000 events were acquired in linear mode; fluores- cence intensities at wavelengths of $530 \mathrm{~nm}$ and $650 \mathrm{~nm}$ were acquired at log-scale. All flow-cytometry data were analysed with the software WinMDI, Version 2.8 (Trotter 1998: http://facs.scripps.edu/software.html). Cellular debris with low FSC characteristics and dead cells (propidium-iodide-positive) were excluded from further evaluation. Different cellular subsets were identified according to their characteristic FSC/SSC profiles. For the assessment of phagocytosis activity after in vitro stimulation, plates with cultured cells were placed on ice (15 min) and briefly shaken, and the whole content of each well was transferred to individual flow-cytometer tubes. Phagocytic active cells were green-fluorescence-positive after ingestion of fluorescent latex beads. For the adjustment of cell numbers for in vitro assays, total cell counts in suspensions of freshly isolated cells were determined with the standard cell dilution assay (SCDA, Pechhold et al. 1994) in a modified form: $2 \times 10^{5}$ green fluorescent standard particles $(4 \mu \mathrm{m}$, Polyscience, USA) were added to each tube. Standard particles (greenfluorescence-positive) could be easily discriminated from viable cells (propidium-iodide-negative, greenfluorescence-negative). Absolute numbers of cells in individual samples were calculated according to $\mathrm{N}$ $($ vital cells $)=$ events $($ vital cells $) \times$ number $($ standard beads)/events (standard beads).

Leukocyte cultivation. For cell-culture experiments, HKL were incubated in 96-well flat-bottomed microtitre plates $\left(2 \times 10^{5}\right.$ cells well $^{-1}$ in a final volume of $175 \mu \mathrm{l})$. All setups were made in duplicate at least. Mitogen stimulation of cultured leukocytes was induced with PWM (pokeweed mitogen, $2 \mathrm{mg} \mathrm{l}^{-1}$ ). Parasite extracts were added at final concentrations of 10 and $100 \mathrm{mg} \mathrm{l}^{-1}$ (protein fraction). The cultures were incubated for $4 \mathrm{~d}$ at $18^{\circ} \mathrm{C}$ in a water-vapour-saturated atmosphere with $2 \% \mathrm{CO}_{2}$.

Production of reactive oxygen species by head kidney leukocytes. Generation of reactive oxygen species (ROS) by HKL was measured by the nitro blue tetrazolium salt (NBT, Sigma-Aldrich) reduction assay. A first set of HKL was collected at Day 40 p.i. and tested immediately after isolation to assess the ex vivo ROS production. Therefore $4 \times 10^{5}$ cells per well of 96 -well flat-bottomed microtitre plates were incubated in $175 \mathrm{\mu l}$ culture medium containing the reactive reagents. A second set of HKL was isolated at Day 48 p.i. and tested for ROS production after in vitro stimulation with Schistocephalus solidus antigens, as described above. Culture supernatants were removed from each well and replaced by $175 \mu \mathrm{l}$ HKL medium containing the respective reactive components. Both HKL setups were tested following the same protocol for the NBTreduction assay. Receptor-independent ROS production was induced by adding $0.15 \mathrm{mg} \mathrm{l}^{-1}$ phorbol myris- 


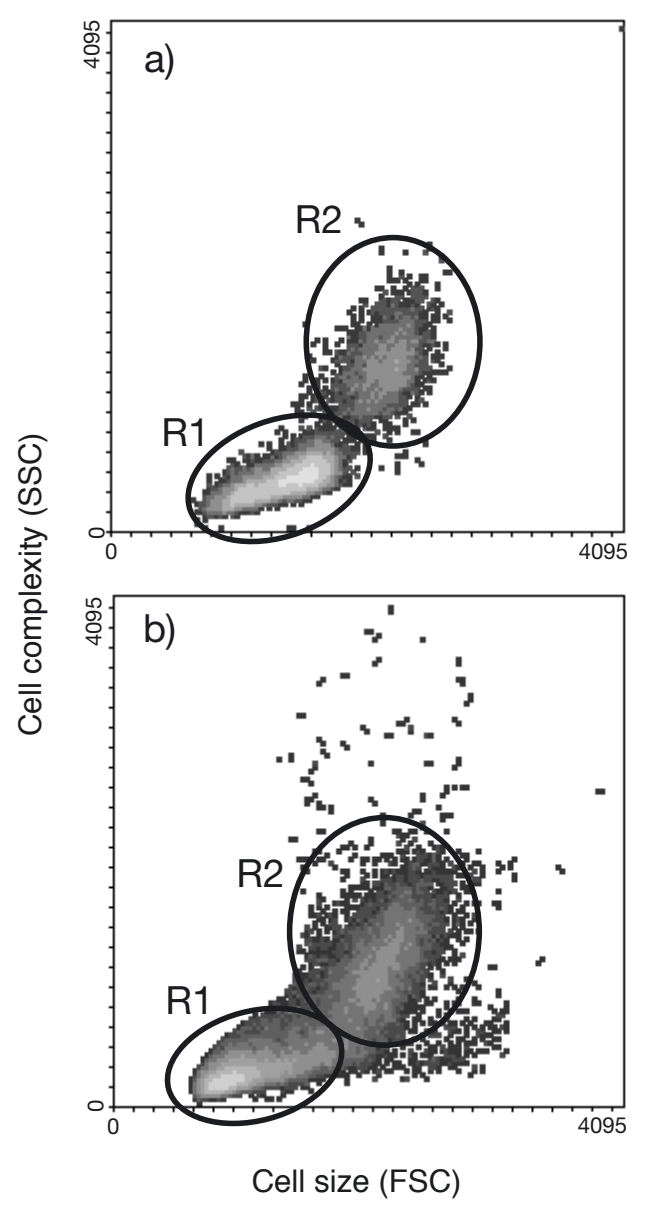

Fig. 1. Density plots showing flow-cytometry characteristics of stickleback leukocytes. In leukocytes isolated from (a) the peripheral blood and (b) the head kidney, 2 distinct cell populations were detected according to their FSC/SSC (forward/side-scatter) profiles. Cells gated in R1 were small lymphocytes, cells gated in R2 were granulocytes

tate acetate (PMA, Sigma-Aldrich). The indicator NBT was added at $1 \mathrm{~g} \mathrm{l}^{-1}$, and wells without PMA served to determine spontaneous ROS generation. After incubation for $2 \mathrm{~h}$ at $22^{\circ} \mathrm{C}$, the supernatants were removed and the cells were fixed by adding $125 \mu$ of $100 \%$ methanol. Each well was washed 2 times with $125 \mu$ of $70 \%(\mathrm{v} / \mathrm{v})$ methanol and the fixed cells were air-dried for $20 \mathrm{~h}$. The reduced NBT (formazan) was dissolved in $125 \mu \mathrm{l} 2 \mathrm{~mol} \mathrm{l}^{-1} \mathrm{KOH}$ and $125 \mu \mathrm{l}$ DMSO per well. Optical densities were recorded with a spectrophotometer at $650 \mathrm{~nm}$.

Phagocytic activity. Phagocytic activity of cultured HKL was tested as described by Chilmonczyk \& Monge (1999). HKL isolated at Day 44 p.i. were cultured as described above for $3 \mathrm{~d}$ in vitro and coincubated with $2.5 \times 10^{6}$ green fluorescent latex particles $(1 \mu \mathrm{m}$, Polyscience, USA) for an additional $18 \mathrm{~h}$. Harvested cells were analysed by flow cytometry for the presence and fraction of green fluorescent cells (phagocytosis-positive).

Statistics. To determine the significance of differences between independent groups, data were compared by ANOVA, and by ANOVA-on-ranks if the equal variance test failed. Results were confirmed using Student's $t$-test, and a Mann-Whitney rank-sum test, if the normality test failed. Differences between dependent groups were tested with a paired $t$-test. Correlation between data sets was tested by regression analysis. All statistical tests were done using Jandel, Sigma Stat 2.0 software.

\section{RESULTS}

\section{Lymphocyte and granulocyte frequencies in blood and head kidney of sticklebacks}

In isolates of stickleback peripheral blood and head kidney leukocytes, 2 distinct cell populations were identified by means of flow cytometry according to their forward-/side scatter characteristics (FSC/SSC profiles; Fig. 1). A population of small cells with lowcomplexity values (FSC/SSC low) was characterised as lymphocytes by parallel microscopic analysis (R1, Fig. 1). The majority of large cells with high complexity (FSC/SSC high), microscopically showed characteristics of granulocytes, such as increased numbers of granules and polymorphic nuclei, and were gated in Region 2 (R2, Fig. 1).

To follow potential changes in frequencies of lymphocytes (R1-cells) and granulocytes (R2-cells) during Schistocephalus solidus infection, leukocytes from the head kidney and blood were analysed by flow cytometry. During the course of infection, increasing proportions of granulocytes and decreasing proportions of lymphocytes were initially observed in the head kidney and blood (Fig. 2a,b). Interestingly, this progression was reversed after $63 \mathrm{~d}$ p.i. and the proportion of granulocytes started to decrease. Development of $S$. solidus plerocercoids did not appear to be affected by the mobilisation of granulocytes, as parasites were growing continuously throughout the observation period up to $98 \mathrm{~d}$ p.i. (Fig. 2c).

\section{Respiratory burst-activity of freshly isolated head kidney leukocytes}

To analyze whether modulation by Schistocephalus solidus plerocercoids could be the cause for the drop in granulocyte mobilisation, a second infection experiment was conducted. Head kidney leukocytes were functionally analysed at time points before the peak 

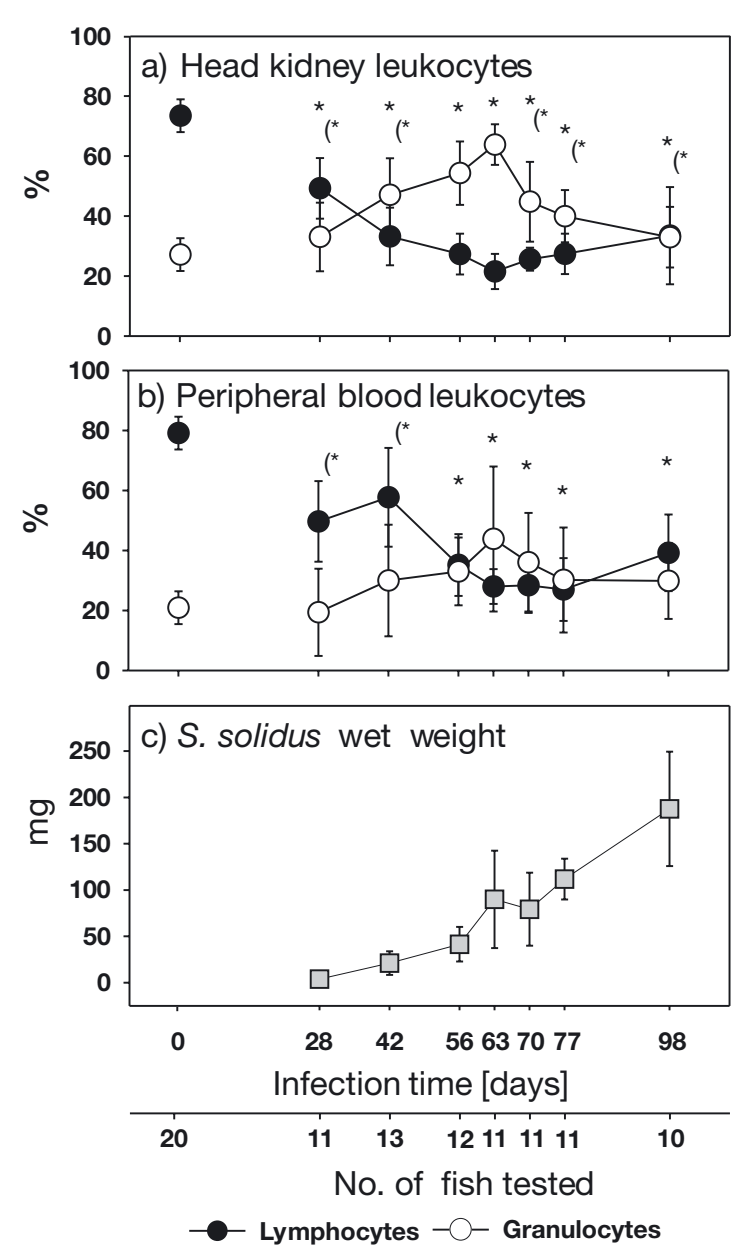

Fig. 2. Gasterosteus aculeatus infected with Schistocephalus solidus. Changes in leukocyte subpopulations in sticklebacks. (a) Head kidney leukocytes and (b) peripheral blood leukocytes were analysed by flow cytometry during (c) course of infection. Proportions of lymphocytes and granulocytes were determined according to the regions shown in Fig. 1. Day 0 measurement represents non-infected fish $(\mathrm{n}=20)$; at subsequent time points, data are mean and $\pm \mathrm{SD}$ of indicated number of infected fish out of 15 fish exposed to the parasite. Note decreasing proportions of granulocytes (R2 cells) after $63 \mathrm{~d}$ p.i. although $S$. solidus were still present and growing. Data on parasite growth (c) were collected by Häberli et al. (2002). *: Significantly different from Day 0; $p<0.05$; *: significantly different from Day 63; $\mathrm{p}<0.05$

granulocyte activity seen in the first infection experiment. When analysed with the NBT-reduction assay immediately after isolation at Day 40 p.i., head kidney leukocytes from infected and from sticklebacks that had cleared the infection (exposed but not infected) exhibited a significantly higher production of reactive oxygen species (ROS) when stimulated with phorbol myristate acetate (PMA) than sham-exposed control fish (exposed to non-infected copepods; Fig. 3). Exposed but non-infected sticklebacks showed an increased spontaneous (not PMA-induced) ROS production (Fig. 3) compared with infected and shamexposed fish.

In infected fish, but not in sham-exposed fish, and exposed but not infected fish, proportions of granulocytes (R2 cells) present in head kidney isolates were significantly correlated to the amount of ROS produced in PMA ( $\mathrm{r}=0.819 ; \mathrm{p}<0.05)$-stimulated cultures and cultures without PMA $(\mathrm{r}=0.794 ; \mathrm{p}<0.05)$. This indicates that higher numbers of granulocytes in head kidney isolates from infected fish, rather than elevated capacities for ROS production of single cells, caused the increased PMA response.

\section{Respiratory burst-activity of head kidney leukocytes after in vitro stimulation}

For further characterisation of the influence of Schistocephalus solidus on head kidney leukocytes, cells were stimulated in culture with $S$. solidus antigens and subsequently tested for production of ROS. Head kidney leukocytes were isolated $48 \mathrm{~d}$ p.i. and incubated in medium alone, with poke weed mitogen (PWM) and with $S$. solidus extracts, and tested in the NBT-

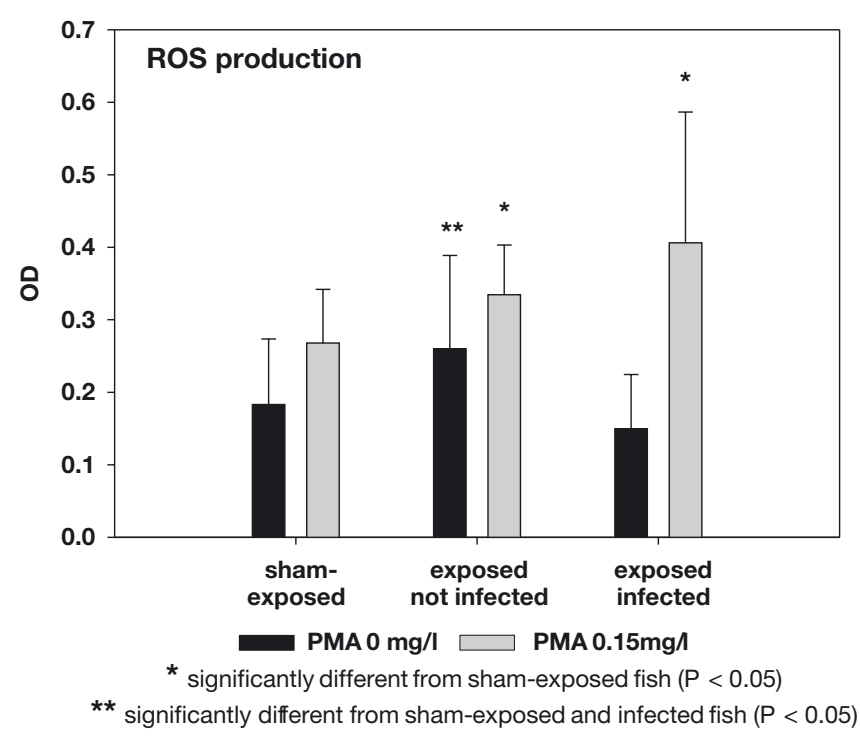

Fig. 3. Respiratory-burst activity of freshly isolated head kidney leukocytes from sticklebacks infected and not infected with Schistocephalus solidus. Production of reactive oxygen species (ROS) was measured immediately after cell isolation (40 d p.i.). Optical density (OD) values are mean \pm SD of triplicate cultures from control $(\mathrm{n}=6)$, exposed but noninfected $(n=5)$ and infected $(n=7)$ fish. Note that head kidney leukocytes from $S$. solidus-infected and -exposed but not infected sticklebacks exhibited a significantly higher phorbol myristate acetate (PMA) response than shamexposed controls. In exposed but non-infected fish, spontaneous (not PMA-induced) ROS production was significantly increased 


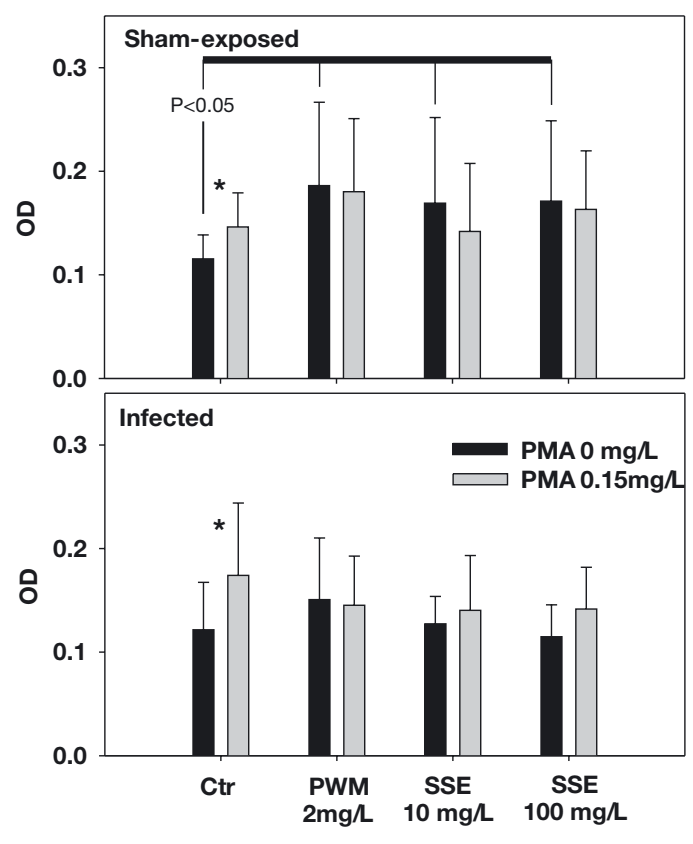

Fig. 4. Gasterosteus aculeatus infected with Schistocephalus solidus. Respiratory-burst activity of head kidney leukocytes after stimulation in cell culture. Head kidney leukocytes from sticklebacks infected $(\mathrm{n}=10)$ and not infected (sham-exposed, $\mathrm{n}=6$ ) with Schistocephalus solidus (48 d p.i.) were cultured in duplicate for $4 \mathrm{~d}$ in medium alone (Ctr), with poke weed mitogen (PWM) and with $S$. solidus extract (SSE). After the incubation period, respiratory-burst activity was measured. Control cultures from both fish groups exhibited an elevated ROS response to stimulation with PMA $(*=p<0.05)$ that was not detectable after previous cultivation with PWM and $S$. solidus extract. While for leukocytes from sham-exposed fish spontaneous (without PMA) ROS production significantly increased after cultivation with PWM and $S$. solidus extract, for leukocytes from infected fish no significant response to cultivation with PWM and $S$. solidus extract was observed

reduction assay. After $4 \mathrm{~d}$ cultivation in medium alone (Ctr; Fig. 4), head kidney leukocytes from shamexposed and S. solidus-infected sticklebacks exhibited a significant ROS response to stimulation with phorbol myristate acetate (PMA). When cultured in medium with PWM or S. solidus extract (SSE; Fig. 4), supplementation with PMA did not result in an elevated ROS production in either setup. Interestingly, leukocytes from sham-exposed fish exhibited a significantly higher spontaneous (not PMA-induced) ROS production in cultures stimulated with PWM and extracts of $S$. solidus than the corresponding control (Fig. 4), indicating an in vitro response to these stimuli (Fig. 4). In contrast, spontaneous ROS production of head kidney leukocytes from infected fish in cultures stimulated with $S$. solidus antigen did not exceed the values recorded for control cultures. Thus head kidney leukocytes from $S$. solidus-infected fish in this assay did not respond to stimulation with $S$. solidus antigens. Data of head kidney leukocytes from exposed but not infected fish $(n=2)$ did not show significant differences between treatment groups (data not shown).

\section{Phagocytosis activity of stickleback head kidney leukocytes after in vitro stimulation}

Comparable observations were made when head kidney leukocytes (isolated at Day 44 p.i.) were analysed for phagocytic activity after $4 \mathrm{~d}$ in vitro stimulation (Fig. 5). Head kidney granulocytes from shamexposed fish significantly increased their phagocytic activity in cultures stimulated with PWM and extracts of $S$. solidus. Granulocytes from infected fish were responding to PWM stimulation, but not to stimulation with $S$. solidus extracts with increased phagocytic activity. Data of head kidney leukocytes from exposed but not infected fish $(\mathrm{n}=2)$ are not shown, as significant differences between treatment groups were not found.

Taken together, Schistocephalus solidus-primed granulocytes ex vivo showed increased capacities for ROS production compared with sham-treated controls (Fig. 3). Cultivation of head kidney leukocytes with $S$. solidus extract triggered in vitro ROS production and

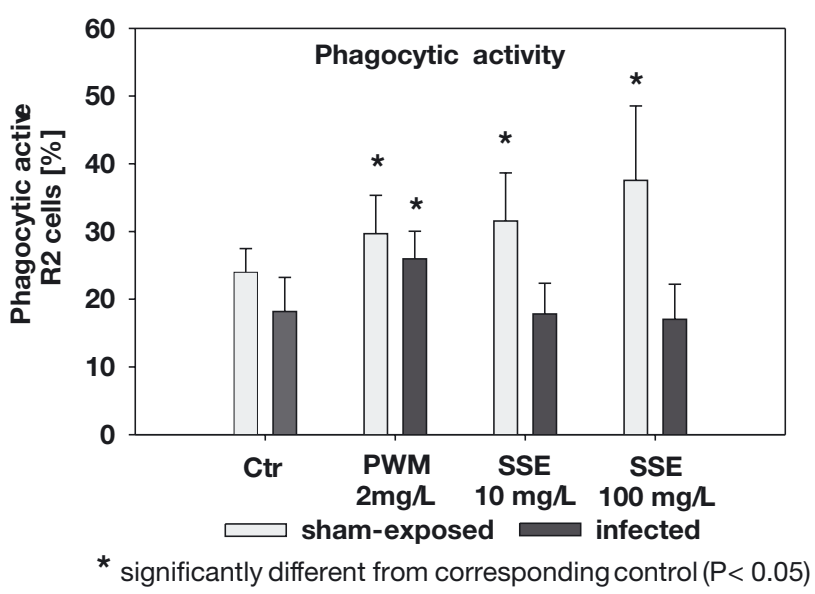

Fig. 5. Gasterosteus aculeatus infected with Schistocephalus solidus. Phagocytic activity of head kidney leukocytes after stimulation in cell culture. Head kidney leukocytes from sticklebacks infected $(\mathrm{n}=9)$ and not infected (sham-exposed, $\mathrm{n}=6$ ) with Schistocephalus solidus (44 d p.i.) were cultured in duplicate for $3 \mathrm{~d}$ in medium alone (Ctr), with PWM (2 $\left.\mathrm{mg} \mathrm{l}^{-1}\right)$ and with $S$. solidus extract (SSE). Fluorescent particles were added for another $18 \mathrm{~h}$ incubation. Proportions of phagocytosis (green fluorescence)-positive granulocytes (R2 cells) were determined by flow cytometry. While head kidney leukocytes from sham-exposed control fish exhibited a significant increase in phagocytic activity in cultures with PWM and extracts of $S$. solidus, infected fish did not respond to stimulation with $S$. solidus antigens, but did respond to stimulation with PWM 
phagocytic activity of cells from sham treated control fish, but leukocytes from infected fish did not respond to direct contact with $S$. solidus extracts (Figs. $4 \& 5$ ).

\section{DISCUSSION}

The present study investigated responses of leukocytes from the stickleback to the pseudophyllidean cestode Schistocephalus solidus. During the initial phase of infection, proportions of granulocytes in leukocyte isolates from the head kidney and blood increased, while the proportions of lymphocytes decreased (Fig. 2a,b). In another study by Barber et al. (2001), elevated counts of total white blood cells were detected in S. solidus-infected G. aculeatus; however, their study did not distinguish between lymphocytes and granulocytes. Mobilisation of granulocytes is a common feature of the immune responses of fishes to helminth parasites (Taylor \& Hoole 1989, Sharp et al. 1992, Secombes \& Chappell 1996). The initial increase in granulocyte proportions in the blood and head kidney of infected stickleback described here could be due to the mobilisation of granulocytes against $S$. solidus rather than to changes in lymphocyte frequencies. Whether the changes in the lymphocyte and granulocyte populations are associated with a leukopenia or leukocytosis remains to be verified, as total leukocyte counts in the blood were not done during the present study. Production of reactive oxygen species (ROS) by head kidney leukocytes from $S$. solidus-infected stickleback in response to stimulation with phorbol myristate acetate (PMA) showed an increase when analysed immediately after isolation (40 d p.i., Fig. 3). This finding is corroborated by another experiment, in which head kidney leukocytes from sticklebacks infected with $S$. solidus showed a higher respiratory-burst reaction upon phagocytosis of zymosan particles in vitro (Kurtz et al. 2004). In the present study, ROS production was positively correlated with proportions of granulocytes present in the head kidney isolates of infected fish, indicating that higher numbers of granulocytes in head kidney isolates rather than elevated capacities for ROS production of single cells caused increased PMA response. Stickleback that had cleared the infection (exposed but not infected) exhibited an increased spontaneous (not PMA-induced) ROS production (Fig. 3) that was not correlated to the proportions of granulocytes in the head kidney isolates, suggesting that single cells had an elevated capacity for ROS production. This was not observed in sticklebacks showing development of $S$. solidus plerocercoids, indicating a differential regulation of granulocyte activation in the groups tested, perhaps suppression by the par- asite. Granulocyte mobilisation and activation obviously did not affect the growth of $S$. solidus plerocercoids, which increased their body weight continuously throughout the observation period (Fig. 2c). In roach infected with the cestode Ligula intestinalis, parasites survive apparently unharmed, despite the presence of a host cellular immune-response, as long as the host is alive (Hoole \& Arme 1982). In the present study, none of the infected or sham-exposed fish died during the experiment. Interestingly, after $63 \mathrm{~d}$ p.i., the proportions of granulocytes started to decrease in the blood and head kidney, while lymphocytes were increasing (Fig. 2). This might reflect the ability of S. solidus to impair the cellular response of its host. To further investigate this assumption, head kidney leukocytes from infected and sham-treated stickleback were isolated before peak granulocyte mobilisation and functionally tested in vitro.

When analysing the ROS production of head kidney leukocytes from infected and sham-exposed fish after cultivation in medium alone (Ctr, Fig. 4), leukocytes from both groups responded to receptor-independent stimulation with PMA, indicating that responsiveness of cells from both groups was still present after the incubation period. In corresponding cultures incubated with PWM and Schistocephalus solidus extract, a further increase in ROS production was not induced by the addition of PMA. This might be due to the fact that, after incubation with PWM and $S$. solidus extract, leukocytes were already triggered for maximum ROS production and were no longer responsive to receptorindependent PMA stimulation. However, head kidney leukocytes from sham-exposed fish showed a significantly higher spontaneous ROS production following in vitro stimulation with PWM and $S$. solidus extracts than medium controls (Fig. 4). In contrast, head kidney leukocytes from $S$. solidus-infected stickleback did not show elevated ROS production in the presence of $S$. solidus extracts. Comparable observations were made when analysing the phagocytic activity of head kidney leukocytes from infected and sham-treated stickleback. While leukocytes from sham-exposed fish showed increased phagocytosis after stimulation with PWM and S. solidus extracts, leukocytes from infected fish responded to PWM but not to $S$. solidus extract. As leukocytes from infected fish showed a significant response to $\mathrm{PWM}$, the lack of response to $S$. solidus extracts was not due to a general anergy of these cells. Nie \& Hoole (2000) used a polarisation assay to test granulocytes responses to Bothriocephalus acheilognathi. In unstimulated controls at $10^{\circ} \mathrm{C}, 98$ to $100 \%$ of granulocytes remained spherical. Addition of parasite extracts and increase in temperature resulted in the formation of lamellipodia at the anterior cell-pole and small tails at the posterior cell-pole. Head kidney gran- 
ulocytes from carp, naturally and laboratory-infected with the cestode $B$. acheilognathi, exhibited a reduced polarisation response to extracts of the parasite compared with granulocytes from naïve carp (Nie \& Hoole 2000). This suppression of polarisation was reduced by addition of carp serum to the in vitro system (Nie \& Hoole 2000). Thus, also in the system used by Nie \& Hoole (2000), a general anergy of leukocytes from infected carp can be excluded. In both systems, cytotoxic effects induced by the parasite extracts are unlikely, as leukocytes from naïve fish clearly responded.

The reduced in vitro responsiveness of leukocytes from infected Gasterosteus aculeatus seems to be part of a complex system. A specific in vivo priming of leukocytes by Schistocephalus solidus plerocercoids seems to be a prerequisite for the in vitro nonresponsiveness to $S$. solidus extracts. For mammals infected with helminth parasites, it has been suggested that granulocyte activity is capable of killing larval, but not adult, parasite stages (Meusen \& Balic 2000). In our experiments, the ongoing growth of $S$. solidus throughout the observation period of $98 \mathrm{~d}$ indicates that once the plerocercoid is developing in the body cavity of $G$. aculeatus, the immune response of the host seems no longer able to repress the parasite completely. In a recent experiment (Kurtz et al. 2004), S. solidus tapeworms reached a relatively smaller size $11 \mathrm{wk}$ p.i. in their stickleback hosts, which possessed an optimal allelic diversity at their major histocompatibility complex (MHC) genes. In stickleback with optimal MHC diversity, a reduced impact of $S$. solidus and other parasites (Wegner et al. 2003a,b) was observed. During ongoing $S$. solidus infection, sticklebacks may be more dependent on mechanisms of acquired immunity and down-regulate functions of the innate immune system. However, mechanisms of immuno-suppression and immuno-avoidance of helminth parasites in fishes are barely understood (Secombes \& Chappell 1996). Hoole \& Arme (1983) suggested that in gudgeon Gobio gobio infected with Ligula intestinalis, protective host proteins are absorbed onto the surface of the parasite. In mammals infected with helminth parasites, pseudocytokines produced by the parasites are suggested to play a role in immune evasion and exploitation (reviewed by Damian 1997). In fishes, factors produced by helminths can influence the immune response. For example, the proliferative response of splenic lymphocytes from roach to mitogens was suppressed in the presence of L. intestinalis extracts (Taylor \& Hoole 1994), and inhibition of temperature-induced polarisation of roach leukocytes occurred with $L$. intestinalisderived extracts (Taylor \& Hoole 1993).

Bråten (1966) observed an ongoing growth of Schistocephalus solidus plerocercoid homotransplants from infected to naïve Gasterosteus aculeatus, but not in heterotransplants to naïve Pungitius pungitius. Heterotransplants of $S$. solidus plerocercoids to other fish species died within 2 to $10 \mathrm{~d}$ (Bråten 1966). Thus mechanisms of immuno-avoidance described in the present study are likely to be a specific feature of the $S$. solidus-G. aculeatus host-parasite relationship. In a few sticklebacks in the present study, no $S$. solidus plerocercoids were found after exposure to the parasite. All copepods used for the exposure were microscopically checked for the presence of $S$. solidus procercoids and were definitely ingested, since no remaining copepods were found in the tanks. Thus, exposed but not infested sticklebacks were able to effectively prevent infective $S$. solidus procercoids from entering the body cavity or eliminate already established plerocercoids. Interestingly, elevated spontaneous ROS production was observed exclusively with leukocytes from exposed but not infected fish (Fig. 3), indicating that production of ROS is indeed part of a successful immune response against $S$. solidus. Infected fish showed a significantly lower spontaneous ROS production, but a high response after PMA stimulation. This might indicate that, once $S$. solidus plerocercoids are growing in the body cavity of $G$. aculeatus, granulocytes are unable to develop their activity against the parasite. Investigations on peritoneal leukocytes isolated during $S$. solidus infection might provide interesting complements to the present work. However, access to leukocytes in sufficient numbers for functional assays is limited in the stickleback. The use of leukocytes from the pronephros is a compromise; however, functional responses of pronephros leukocytes from infected and non-infected stickleback are differential, reflecting an in vivo priming by the parasite.

Schistocephalus solidus appears to be very well adapted to the immunological features of its specific intermediate host Gasterosteus aculeatus. The initial phase of infection seems to be decisive for the development of a parasitosis. It is likely that mobilisation and activation of granulocytes can be effective against procercoid stages of $S$. solidus during the first weeks of infection, but once plerocercoid stages are present in the body cavity, granulocytes are unable to develop their activity against $S$. solidus.

Acknowledgements. We would like to thank P. B. Aeschlimann, M. A. Häberli and M. Michaud for their support in the infection experiments. Furthermore, we thank N. Karstens for her help in flow-cytometer trouble-shooting. We are grateful to G. F. Wiegertjes, Cell Biology and Immunology Group, Wageningen University, The Netherlands, for critical reading of the manuscript. 


\section{LITERATURE CITED}

Aeschlimann P, Häberli M, Milinski M (2000) Threat-sensitive feeding strategy of immature sticklebacks (Gasterosteus aculeatus) in response to recent experimental infection with the cestode Schistocephalus solidus. Behav Ecol Sociobiol 49:1-7

Arme C, Owen RW (1967) Infections of the three-spined stickleback, Gasterosteus aculeatus L., with the plerocercoid larvae of Schistocephalus solidus (Müller, 1776), with special reference to pathological effects. Parasitology 57: 301-14

Arnott SA, Barber I, Huntingford FA (2000) Parasite-associated growth enhancement in a fish-cestode system. Proc R Soc Lond B 267:657-63

Barber I, Ruxton GD (1998) Temporal prey distribution affects the competitive ability of parasitized sticklebacks. Anim Behav 56:1477-1483

Barber I, Arnott SA, Braithwaite VA, Andrew J, Huntingford FA (2001) Indirect fitness consequences of mate choice in sticklebacks: offspring of brighter males grow slowly but resist parasitic infections. Proc R Soc Lond B 268:71-76

Binz T, Reusch TBH, Wedekind C, Schärer L, Sternberg JM, Milinski M (2000) Isolation and characterization of microsatellite loci from the tapeworm Schistocephalus solidus. Mol Ecol 9:1926-1927

Bråten T (1966) Host specificity in Schistocephalus solidus. Parasitology 56:657-664

Candolin U, Voigt HR (2001) No effect of a parasite on reproduction in stickleback males: a laboratory artefact? Parasitology 122:457-464

Chilmonczyk S, Monge D (1999) Flow cytometry as a tool for assessment of the fish cellular immune response to pathogens. Fish Shellfish Immunol 9:319-333

Christen M, Milinski M (2003) The consequence of self-fertilization and out-crossing of the cestode Schistocephalus solidus in its second intermediate host. Parasitology 126: 369-378

Damian RT (1997) Parasite immune evasion and exploitation: reflections and projections. Parasitology 115(Suppl): S169-S175

Dubinina MN (1980) Tapeworms (Cestoda, Ligulidae) of the fauna of the USSR. Amerind Publishing, New Delhi

Häberli MA, Aeschlimann PB, Michaud M, Milinski M (2002) Growth pattern of infection and sex bias to prevalence of Schistocephalus solidus larvae in three-spined sticklebacks (Gasterosteus aculeatus). In: Häberli MA, Odour cues and sexual selection in the three-spined stickleback (Gasterosteus aculeatus). PhD thesis, University of Berne

Hoole D, Arme C (1982) Ultrastructural studies on the cellular response of roach, Rutilus rutilus L., to the plerocercoid larva of the pseudophyllidean cestode, Ligula intestinalis. J Fish Dis 5:131-144

Hoole D, Arme C (1983) Ultrastructural studies on the cellular response of fish hosts following experimental infection with the plerocercoid of Ligula intestinalis (Cestoda: Pseudophyllidae). Parasitology 87:139-149

Kurtz J, Kalbe M, Aeschlimann PB, Häberli MA, Wegner KM, Reusch TBH, Milinski M (2004) Major histocompatibility complex diversity influences parasite resistance and innate immunity in sticklebacks. Proc R Soc Lond B 271:197-204

McPhail JD, Peacock SD (1983) Some effects of the cestode Schistocephalus solidus on reproduction in the threespined stickleback Gasterosteus aculeatus, evolutionary aspects of host-parasite interaction. Can J Zool 61: 901-908
Meakins RH (1974) A quantitative approach to the effects of the plerocercoid of Schistocephalus solidus Müller 1776 on the ovarian maturation of the three-spined stickleback Gasterosteus aculeatus L. Z Parasitenkd 44:73-79

Meusen EN, Balic A (2000) Do eosinophils have a role in the killing of helminth parasites? Parasitol Today 16:95-101

Milinski M (1984) Parasites determine a predator's optimal feeding strategy. Behav Ecol Sociobiol 15:35-37

Milinski M (1985) Risk of predation of parasitized sticklebacks (Gasterosteus aculeatus) under competition for food. Behaviour 93:203-216

Milinski M (1990) Parasites and host decision making. In: Barnard CJ, Behnke JM (eds) Parasitism and host behaviour. Taylor \& Francis, London, p 95-116

Miller NW, McKinney EC (1994) In vitro culture of fish leukocytes. In: Hochachka PW, Mommsen TP (eds) Biochemistry and molecular biology of fishes. Elsevier Science, Amsterdam, p 341-353

Nie P, Hoole D (2000) Effects of Bothriocephalus acheilognathi on the polarization response of pronephric leucocytes of carp, Cyprinus carpio. J Helminthol 74:253-257

Orr TSC, Hopkins CA, Charles GH (1969) Host specificity and rejection of Schistocephalus solidus. Parasitology 59: 683-690

Øverli O, Pall M, Borg B, Jobling M, Winberg S (2001) Effects of Schistocephalus solidus infection on brain monoaminergic activity in female three-spined sticklebacks Gasterosteus aculeatus. Proc R Soc Lond B 268:1411-1415

Pechhold K, Pohl T, Kabelitz D (1994) Rapid quantification of lymphocyte subsets in heterogeneous cell populations by flow cytometry. Cytometry 16:152-159

Reimchen TE, Nosil P (2001) Ecological causes of sex-biased parasitism in threespine stickleback. Biol J Linn Soc 73: $51-63$

Secombes C J, Chappell LH (1996) Fish immune responses to experimental and natural infection with helminth parasites. Annu Rev Fish Dis 6:167-177

Sharp GJ, Pike AW, Secombes CJ (1992) Sequential development of the immune response in rainbow trout (Oncorhynchus mykiss [Walbaum, 1792]) to experimental plerocercoid infections of Diphyllobothrium dendriticum (Nitzsch, 1824). Parasitology 104:169-178

Smyth JD (1954) Studies on tapeworm physiology. VII. Fertilization of Schistocephalus solidus in vitro. Exp Parasitol 3: $64-71$

Taylor MJ, Hoole D (1989) Ligula intestinalis (L.) (Cestoda: Pseudophyllidea): plerocercoid-induced changes in the spleen and pronephros of roach, Rutilus rutilus (L.) and gudgeon, Gobio gobio (L.). J Fish Biol 34:583-596

Taylor MJ, Hoole D (1993) Ligula intestinalis (L.) (Cestoda: Pseudophyllidea): polarization of cyprinid leucocytes as an indicator of host- and parasite-derived chemoattractants. Parasitology 107:433-440

Taylor MJ, Hoole D (1994) Modulation of fish lymphocyte proliferation by extracts and isolated proteinase inhibitors of Ligula intestinalis (Cestoda). Fish Shellfish Immunol 4: 221-230

Taylor MJ, Hoole D (1995) The chemiluminescence of cyprinid leucocytes in response to zymosan and extracts of Ligula intestinalis (Cestoda). Fish Shellfish Immunol 5:191-198

Wedekind C, Strahm D, Schärer L (1998) Evidence for strategic egg production in a hermaphroditic cestode. Parasitology 117:373-382

Wegner KM, Kalbe M, Kurtz J, Reusch TBH, Milinski M (2003a) Parasite selection for immunogenetic optimality. Science 30:1343

Wegner KM, Reusch TBH, Kalbe M (2003b) Multiple para- 
sites are driving major histocompatibility complex polymorphism in the wild. J Evol Biol 16:233-241

Whyte SK, Chappell LH, Secombes CJ (1989) Cytotoxic reactions of rainbow trout, Salmo gaidneri Richardson, macrophages for larvae of the eye fluke Diplostomum

Editorial responsibility: Wolfgang Körting,

Hannover, Germany spathaceum (Digenea). J Fish Biol 35:333-345

Whyte SK, Chappell LH, Secombes CJ (1990) Protection of rainbow trout (Salmo gaidneri) against Diplostomum spathaceum (Digenea): the role of specific antibody and activated macrophages. J Fish Dis 13:281-291

Submitted: June 30, 2003; Accepted: November 10, 2003

Proofs received from author(s): April 21, 2004 